OPEN ACCESS

Edited by:

Michael Patrick Schaub,

University of Zurich, Switzerland

Reviewed by:

Michael Musker,

University of South Australia, Australia

Jin Han,

University of New South

Wales, Australia

*Correspondence:

Patrick Dülsen

patrick.duelsen@uni-ulm.de

Specialty section:

This article was submitted to

Digital Mental Health,

a section of the journal

Frontiers in Digital Health

Received: 16 November 2021

Accepted: 19 January 2022

Published: 16 February 2022

Citation:

Dülsen P, Barck K, Daubmann A,

Höller A, Zeidler J, Kilian $R$

Wiegand-Grefe $S$ and Baumeister $H$ (2022) Clinical- and Cost Effectiveness

of a Guided Internet-Based

Intervention for Children (12-18 Years)

of Parents With Mental Disorders

(iCHIMPS): Study Protocol of a

Multicentered Cluster-Randomized

Controlled Trial.

Front. Digit. Health 4:816412.

doi: $10.3389 /$ fdgth.2022.816412

\section{Clinical- and Cost Effectiveness of a Guided Internet-Based Intervention for Children (12-18 Years) of Parents With Mental Disorders (iCHIMPS): Study Protocol of a Multicentered Cluster-Randomized Controlled Trial}

\author{
Patrick Dülsen ${ }^{1 *}$, Katja Barck ${ }^{1}$, Anne Daubmann ${ }^{2}$, Alexandra Höller², Jan Zeidler ${ }^{3}$, \\ Reinhold Kilian ${ }^{4}$, Silke Wiegand-Grefe ${ }^{5}$ and Harald Baumeister ${ }^{1}$ \\ ${ }^{1}$ Department of Clinical Psychology and Psychotherapy, Institute of Psychology and Education, Ulm University, Ulm, \\ Germany, ${ }^{2}$ Department of Medical Biometry, Institute for Medical Biometry and Epidemiology, University Medical Center \\ Hamburg-Eppendorf, Hamburg, Germany, ${ }^{3}$ Center for Health Economics Research Hannover (CHERH), Leibniz University \\ Hannover, Hanover, Germany, ${ }^{4}$ Department of Psychiatry II, BKH Günzburg, Ulm University, Ulm, Germany, ${ }^{5}$ Department of \\ Child and Adolescent Psychiatry, Psychotherapy and Psychosomatics, University Medical Centre Hamburg-Eppendorf, \\ Hamburg, Germany
}

Introduction: Children of parents with mental disorders have a high chance of developing a mental disorder themselves. However, this at-risk group is regularly overlooked and typically not seen by any mental health professionals. Internet- and mobile-based interventions (IMIs) can provide a means of promoting mental health for children of parents with mental disorders.

Objective: The introduced study will evaluate the clinical- and cost-effectiveness of the iCHIMPS IMI in promoting mental health for children of parents with mental disorders.

Methods: A two-armed multicentered cluster-randomized controlled trial (CRCT) comparing the clinical- and cost-effectiveness of the $\mathrm{iCHIMPS} \mathrm{IMI} \mathrm{in} \mathrm{the} \mathrm{intervention}$ group (IG) to a treatment-as-usual (TAU) control group will be conducted. Recruitment will be handled at currently 21 adult mental health clinics throughout Germany. Participating families will be randomly divided into the two groups until the final sample size of 306 participating adolescents (age 12-18) has been reached. The adolescents in the intervention group will receive access to the $\mathrm{IMI}$ and can take part in up to eight intervention modules. Assessment will be conducted during the recruitment (baseline), 1-month, 2-months, and 6-month post-inclusion. Primary outcome is the mental health of the participating adolescents at 6-months post-inclusion as measured by the Youth Self Report score. Secondary self-report outcomes are mental wellbeing, self-efficacy, coping strategies and negative effects as well as mental health of the adolescents as reported by their parent(s). Included moderators are sociodemographic characteristics, working alliance, social support and the mental health diagnoses of the parents. Statistical analyses will be conducted on the intention-to-treat principle as well as with additional per-protocol analyses. Additionally, the cost-effectiveness as well as qualitative data concerning the adherence, acceptance, and feasibility of the IMI will be analyzed. 
Discussion: The iCHIMPS cRCT examines the clinical- as well as cost-effectiveness of the iCHIMPS mental health promotion IMI for children of parents with mental disorders. This provides the opportunity to gain insights into an innovative as well as time- and location-independent form of support for this often-overlooked at-risk group. Additionally, the larger CHIMPS-NET project allows comparisons between internet-based and face-to-face interventions for a similar target group.

Clinical Trial Registration: www.ClinicalTrials.gov, identifier: DRKS00025158.

Keywords: e-mental-health, psychotherapy, children and adolescents, parents with mental disorders, internetand mobile-based intervention

\section{INTRODUCTION}

An estimated $25 \%$ of children and adolescents throughout Germany have at least one parent with a mental disorder (1). The chances of developing any mental disorder for the children themselves have been reported to be as high as $50 \%$, and $33 \%$ for severe mental disorders (2). Wiegand-Grefe and colleagues (3) highlighted a 3-7 times higher risk of showing symptoms of any mental disorder when compared to the general population. Children of parents with a mental disorder, however, are typically not seen by mental health professionals $(4,5)$. One reason for the limited health care coverage might result from the lack of mental health promotion programs specifically tailored to the needs and demands of this at-risk group (6).

Reasons for the vulnerability to mental disorders of children and adolescents with parents with mental disorders are reflected by the complex and heterogenous psychosocial, bio-genetic as well as medical situation of every single family member $(5,7)$. For

Abbreviations: AOK; Allgemeine Ortskrankenkasse; APOI; Attitudes toward Psychological Online Interventions Questionnaire; AUC; area under the curve; Brief COPE; Brief Coping Orientation to Problems Experienced Inventory; CAMSHRI-EU; Children and Adolescent Mental Health Services Receipt Inventory; CBCL; Child Behavior Checklist; CBT; cognitive behavioral therapy; CHIMPS-NET; children of mentally ill parents - network; CONSORT; Consolidated Standards of Reporting Trials; CHEERS; Consolidated Health Economic Evaluation Reporting Standards Statement; CHERH; Center for Health Economic Research in Hannover; cRCT; cluster-randomized controlled trial; DAK; Deutsche Angestellten Krankenkasse; DSM; Diagnostic and Statistical Manual of Mental Disorders; EQ-5D-Y; EuroQol-5 Dimensionen-Youth; GBA; Gemeinsamer Bundesausschuss; ICD; International Statistical Classification of Diseases and Related Health Problems; ICC; intra-cluster correlation coefficient; ICER; incremental cost-effectiveness ratio; iCHIMPS; internet-based intervention for children and adolescents of mentally ill parents; IG; intervention group; IKK Classic; Innungskrankenkasse Classic; IMIs; internet- and mobilebased interventions; ISPOR; International Society for Pharmacoeconomics and Outcomes Research; ITT; intention-to-treat; KIDDI-SADS-PL; Kiddie - Schedule for Affective Disorders and Schizophrenia - Present and Lifetime Version; KKH; Kaufmännische Krankenkasse; MDR; Medical Device Regulation; M.I.N.I.; Mini International Neuropsychiatric Interview; MKK; Mobil Krankenkasse; NEQ; Negative Effects Questionnaire; OSSQ; Oslo Social Support Questionnaire; PP; per protocol; QALY; quality adjusted life year; RCI; reliable change index, (S)AE; (serious) adverse event; SAS; Skala zur Allgemeinen Selbstwirksamkeitserwartung; SSRMI; Self-Stigma in Relatives of People with Mental Illness; TAU; treatment as usual; TK; Techniker Krankenkasse; UKE; Universitätsklinikum Hamburg Eppendorf; WAI; Working Alliance Inventory; WAI-I; Working Alliance Inventory for guided internet interventions; WHO; World Health Organization; WHO-5; Wohlbefindens-Index; YSR; Youth Self Report. example, the parent with a mental disorder can have diagnoses ranging across the whole spectrum of mental disorders. Their partner can either be healthy, on the threshold of developing a mental disorder, mentally ill themselves or mentally ill but not yet diagnosed. On the other hand, the children can be either resilient and not symptomatic, they can be on the threshold or they can already show clinically relevant mental symptoms themselves (7). The huge amount of possible multi-way interactions of these risk- and resilience-factors demonstrate the challenges as well as opportunities posed to the care of such children.

Treatment and prevention approaches for mental disorders based on psychotherapeutic techniques are effective interventions for adults as well as children and adolescents (8-12). Preventive interventions focusing on families with at least one parent with a mental disorder seem to be capable to effectively reduce the risk of developing a mental disorder for the children within these families by up to $40 \%$ (13). However, most of the available interventions target the parents and are therefore not readily suitable for the children themselves, leaving almost no specific interventions for children (6). An additional reason for children of parents with a mental disorder not receiving the professional help they might need is the often existing mental health care gap $(14,15)$ between people in need of care and available programs. It has further been argued that this gap as well as the quality and specificity of available psychotherapeutic interventions can be even more limited for children and adolescents (16). A potential approach to decrease this gap might be given in the form of Internet- and mobile based self-help interventions (IMIs) $(17,18)$. Especially the younger generation with their generally high digital affinity might be more open to use these kinds of mental health promotion interventions.

IMIs can be characterized by their mode of delivery e.g., via webpages or mobile apps and often consist of persuasive features such as videos, audios, and chat functions (19). Furthermore, IMIs are usually grounded on an evidence-based theoretical background, most often cognitive behavioral therapy, but also other approaches such as psychodynamic therapy are available. Additionally, the amount of human support provided by IMIs may vary $(17,20)$. The benefits of internet-based interventions, such as their time- and location-independent form of delivery as well as the personnel-efficient procedure, can increase the amount of mental health promotion programs that are provided to the population (17). There is already substantial evidence 
for the effectiveness of IMIs for children and adolescents who suffer from mental disorders [e.g., $(18,21,22)]$. Evidence of the effectiveness of IMIs for children of parents with a mental disorder is scarce but promising (23-25). Reupert and colleagues (24) reported a reduction in depression and stress scores while another group of researchers stated an improvement in coping and mastery mechanisms after participating in their IMI (25).

Meeting the aforementioned complex needs of families with at least one parent with a mental disorder is the main goal of the joint multicenter research project CHIMPS-NET (CHIldren of Mentally ill ParentS - NETwork). The research project investigates the possibilities of breaking through the circle of transgenerational transmission of mental disorders. For this purpose, three face-to-face prevention and treatment interventions based on the CHIMPS-program (26) are already being evaluated. The present study will evaluate the clinicalas well as the cost-effectiveness of iCHIMPS, a mental health promotion IMI. The intervention was specifically developed for adolescents with parents that have a mental disorder and is generically designed to be used by adolescents who either already show symptoms of mental disorders themselves or not. The overarching goal of the internet-based intervention is the promotion of mental health and the improvement of selfmanagement abilities among adolescents (age 12-18 years). In detail the present trial will examine the following:

1 The clinical-effectiveness of iCHIMPS compared to TAU (treatment-as-usual) regarding mental health at 6-months follow-up

2 The clinical-effectiveness of iCHIMPS compared to TAU (treatment-as-usual) regarding the secondary outcomes (mental-wellbeing, further mental health outcomes, self-efficacy and coping strategies)

3 The cost-effectiveness of providing iCHIMPS in addition to TAUvs. TAU alone

4 Quantitative and qualitative information about the adherence and acceptance as well as potential barriers and facilitators of iCHIMPS

5 Moderators and mediators of intervention success as well as potential adverse events.

\section{METHODS}

\section{Study Design}

A two-armed multicentered cluster-randomized controlled trial (cRCT) comparing the clinical- and cost-effectiveness of the iCHIMPS IMI in the intervention group (IG) to a treatment-asusual (TAU) control group will be conducted.

The study has been approved by the Ethics Committee of Ulm University, Germany (189/20-FSt/bal.) and has been registered in the German clinical trial register (DRKS00025158). Results will be reported according to the Consolidated Standards of Reporting Trials (CONSORT) Statement 2010 and the extensions for reporting cluster randomized trials and trials on psychological interventions $(27,28)$. The cost-effectiveness analyses will be reported in accordance with the Consolidated Health Economic Evaluation Reporting Standards Statement (29), as well as with the guidelines of the International Society for Pharmacoeconomics and Outcomes Research (30).This trial protocol was created according to SPIRIT guidelines (31).

\section{Participants Cluster Definition}

The study population are families, which are defined as (a) having at least one parent with a mental disorder according to the ICD10 [mental disorder is defined by a mental disorder diagnosis made by a licensed health care professional (i.e., physician or psychotherapist) within the last 6 months] as well as (b) at least one child between the age of 12 years and 0 months to 18 years and 11 months.

Additionally, all participating family members (at least the parent with the mental disorder as well as one child) need to (c) have access to a computer, laptop or smartphone as well as to the internet, and have sufficient proficiency in German.

Whole families as a cluster and not the separate family members will be randomized, due to the possibility that siblings from the same family could be randomized into different study groups. Siblings in two different study groups would potentially increase the risk of group contamination and thereby compromise the validity of the study results.

The trial focuses on the children and therefore the parents do not necessarily have to participate in the study, except for giving informed consent and providing information about their mental disorder at the baseline assessment, and the children do not necessarily have to live with their parent(s) or the parent(s) do not necessarily have to have custody of the participating children. For a better readability, the participating children will be referred to as participants hereafter, while their parent(s) and if applicable legal guardian(s) will be called parents.

\section{Inclusion and Exclusion Criteria of Participating Family Members \\ Participants}

All participants need to (a) have signed the informed consent [if the participating adolescent is below 16 years of age, signed informed consent by the parent(s) or, if different custody arrangements apply then the legal guardian(s)] and (b) have successfully completed the baseline assessment.

Exclusion criteria are (a) acute suicidal tendencies, (b) acute substance use disorder (ICD F1X.2 except nicotine dependence F17.2) or (c) acute psychotic symptoms exhibited by the participating adolescent as assessed by clinical professionals at the participating clinics at baseline.

\section{Parent(s) and/or Legal Guardian(s)}

At least one parent with a mental disorder needs to have (a) signed the informed consent and (b) taken part in the baseline assessment.

Further participating family members need to have (a) signed the informed consent.

\section{Further Participation in the Assessments}

The parent(s) and if applicable the legal guardian(s) will be encouraged to take part in the whole assessment procedure, however, their non-participation, apart from the baseline assessment, will not be considered an exclusion criterion. 


\section{Sample Size and Power Calculations}

The sample size calculation was conducted with the Power Analysis and Sample Size Software (PASS 15) and is approximated based on the "Tests for Two Means in a Cluster-Randomized Design" module. As clinically significant mental health effect a moderate effect size of 0.5 (Cohens d) in the primary outcome (Youth Self Report score; YSR 11-18R; 32) between the two groups (iCHIMPS vs. TAU) at 6-months post inclusion has been assumed. Calculating with a power of $80 \%$, a significance level of $0.5 \%$ (two-sided hypothesis), an inter-cluster correlation coefficient of $5 \%$, and a family size of 1.3 children between the age of 12 years and 0 months and 18 years and 11 months per family, 65 participants in 50 families per group (130 participants in 100 families in total) are needed. Considering a drop-out rate for online-interventions of 50-60\%, our trial will aim at 306 participants in 234 families (153 participants in 117 families per group) as has been estimated in the original grant application.

\section{Recruitment}

The recruitment has started on May $1^{\text {st }} 2021$ and will be handled at 21 adult mental health clinics across Germany. Adult mental health clinics have been chosen as recruitment sites due to the fact that children of parents with a mental disorder are regularly overlooked for prevention or treatment offers and are therefore often not or not yet seen by any mental health professional, hence not reachable via the standard mental health care system for children and adolescents. Study employees at the participating adult mental health clinics will additionally recruit potential families from other mental health care locations or through advertising.

\section{Randomization}

Participating family-clusters will be allocated in a 1:1 ratio to either iCHIMPS or TAU group according to a central randomization list based on block wise randomization with a variable block length and without stratification. The randomization list was generated by the Institute of Medical Biometry and Epidemiology of the University Medical Center Hamburg-Eppendorf using the statistical software $\mathrm{R}$ version 3.6.3.

\section{Blinding and Masking}

The randomization as well as the final statistical analysis will be conducted by scientists at Ulm University not otherwise involved in the present trial. The study team, which designed the intervention and assessment, will not be blinded toward participant features or group memberships. Reasons for this approach are feasibility limits and the offered support via email given to the participants. However, since the main risk is posed by unblinded randomization and statistical analysis, we are confident the selected approach is well suited to deliver unbiased results.

\section{Procedure}

At each participating adultmental health clinic an employee, who is briefed about the study, recruits potential families via mental health care locations or advertisements (see Figure 1 for a flow chart representation).

If the potential family meets the inclusion criteria for families and all necessary parties have signed the informed consent as well as the contact forms, a clinical assessment interview will take place, either at the clinics or via phone. The inclusion and exclusion criteria will be written down and transferred to the study team at Ulm University together with the other forms. This marks the end of the recruitment procedure at the adult mental health clinics.

After the recruitment at the clinics is finalized and the inclusion criteria are fulfilled, the participants and possibly their parents and/or legal guardian(s) will receive a personalized link for the online assessment. After the successful completion of the online baseline assessment ( 0 ) by at least the participant and the parent with the mental disorder, the family is eligible to be included into the trial and will be randomized according to the randomization list by a scientist at Ulm University.

Depending on the group the family was randomized to, an email will contain the explanation of the procedure of the internet-based intervention and the following online assessments (iCHIMPS) or the explanation of why they will not receive access to the internet-based intervention and the procedure of the following online assessments (TAU).

If the participant was assigned to iCHIMPS, he or she is able to log into the internet-based intervention at https://patient.esanotrainings.de and complete the eight consecutive modules of the internet-based intervention at her or his own pace.

All participants (ICHIMPS and TAU) and possibly their parent(s) and/or legal guardian(s) will receive emails with an invitation to the online assessments for $\mathrm{t} 1$ (one-month postinclusion), for $\mathrm{t} 2$ (two-months post-inclusion) and for $\mathrm{t} 3$ (sixmonths post-inclusion).

\section{Intervention}

The internet-based intervention iCHIMPS was developed by the study team of the Department of Clinical Psychology and Psychotherapy at Ulm University and builds onto previously devised internet-based interventions of the department (33) as well as on the CHIMPS-program (26). It is a generically designed intervention suitable for children and adolescents, with a parent having a mental disorder, who either already show symptoms of mental disorders themselves or not. The intervention was designed in such a way that it can be carried out by the children and adolescents without any help by an adult, meeting the needs of the potentially complex and strained family situations. The goals of iCHIMPS are to enable children of parents with a mental disorder to be able to better deal with their difficult life situation, as well as to increase their mental health, quality of life and self-management abilities. 
TABLE 1 | Overview of intervention modules.

\begin{tabular}{|c|c|c|}
\hline \multicolumn{2}{|c|}{ Modules } & \multirow{2}{*}{$\begin{array}{l}\text { Content } \\
\text { Introducing the team, literature and guiding principles; Familiarising with the } \\
\text { iCHIMPS process, overview of the modules and E-Coaching }\end{array}$} \\
\hline 1 & What can you expect? & \\
\hline 2 & The first step - get to know yourself and your challenges. & $\begin{array}{l}\text { Strengths and worries of the participants; Identifying one's values, goals and } \\
\text { resources; Challenges in everyday life with a mentally ill parent }\end{array}$ \\
\hline 3 & Knowledge means strength - find out more about your parent's mental illness. & Psychoeducation; Family coping with illness \\
\hline 4 & Talk about it - stay in contact and learn to talk about difficult subjects. & $\begin{array}{l}\text { Relevance of communication, rules for successful communication, talking } \\
\text { about your parent's mental illness with different groups of people }\end{array}$ \\
\hline 5 & Find inner peace - learn how to cope with stress. & Stress management; Possibilities for coping with stress \\
\hline 6 & The world of emotions. & $\begin{array}{l}\text { Explaining Emotions; Coping with negative Feelings, promoting pleasant } \\
\text { feelings }\end{array}$ \\
\hline 7 & You, yourself and your rough edges - learn how to set limits. & Setting limits; Strengthen your self-esteem \\
\hline 8 & Last stop! - What is next? & Summary of Modules 2-7; Recognition of warning signs and prevention \\
\hline
\end{tabular}

iCHIMPS is designed as a series of eight consecutive modules. The content is delivered in text, picture, video as well as audio format and can be partly customized to the individual interests of the user. iCHIMPS is based on four main principles: (1) The intervention is internet and mobile based and can be accessed from any computer, laptop or smartphone, thereby being optimized for devices with larger screens and keyboards. (2) To increase the adherence of the users the design follows persuasive principles (19) and motivational messages as well as sending reminders regularly. Furthermore, after each session a fixed date will be arranged on when the next module will be started. (3) It is based on an evidence based theoretical background combining cognitive behavioral and psychodynamic therapy approaches $(26,33)$. (4) To facilitate the transfer of what the participants have learned during the intervention into their daily lives, interactive home tasks and helpful suggestions in terms of take-home-messages will be provided. Some of the home-tasks can be done together with other family members.

The main topics of the intervention are the engagement with the users own life situation, their own challenges, resources and strengths as well as the engagement about the mental disorder of their parent(s). Further covered topics include health, communicating about difficult topics, strengthening familial and social relationships, stress management, emotion regulation and establishing healthy boundaries to increase autonomy (an overview of the modules is provided in Table 1). The techniques used are based on cognitive behavioral therapy, such as cognitive restructuring, on mindfulness-based therapy as well as psychodynamic therapy, such as clarifying their own life situation and careful reflection about dysfunctional communication or relationship patterns.

Additionally, the internet-based intervention can be complemented by a varying amount of human support. E-Coaches can provide guidance through individualized feedback on each section and answer potential questions in an asynchronous fashion. E-Coaches in the present study are children and adolescents' psychotherapists (in training) who undergo a specialized workshop and regular supervision.

\section{Intervention Platform}

iCHIMPS was designed and will be made accessible on the ehealth platform eSano, an open source platform developed by the Department of Clinical Psychology and Psychotherapy and the Institute of Databases and Information Systems at Ulm University (34). The platform consists of three parts: (1) the content management system, through which the intervention can be designed; (2) the E-coach platform, through which the case management, i.e., process monitoring, guidance and feedback on individual modules can be provided; and (3) the patient application (web-app) through which the participants can access the intervention. All three parts of the platform are located on secured servers and regulated by the necessary safety rules and network security devices. eSano was developed according to the requirements of the German Medial Devices Act and the Medical Device Regulation (MDR), thereby, considering the IEC 62304 (safety class B), the GAMP5 (category 4), the General Principles of Software Validation of the FDA as well as the Pharmaceutical Inspection Cooperation Scheme (PIC/S) 11-3.

\section{Assessments}

The Assessment will be conducted with the participants and at least partly with the parent(s) and/or legal guardian(s) as online self-report questionnaires (via the platform Lime Survey), as in-person or telephone-based interviews, and by the collection of claims data through the participating health insurance companies. An overview of all assessment materials for t0 (baseline assessment), t1 (inter-session assessment 1-month post-inclusion), t2 (2-months post-inclusion) as well as t3 (6-months postinclusion) is provided in Table 2 and will be individually introduced below.

\section{Primary Outcome}

The primary outcome is mental health of the participant at 6-months post-inclusion ( $\mathrm{t} 3$ ) as measured by the Youth Self Report score (YSR 11-18R; 32). All 112 items will be combined to calculate a total problems score. A t-value above 
TABLE 2 | Overview assessment.

\begin{tabular}{|c|c|c|c|c|c|}
\hline \multirow[t]{2}{*}{ Instrument } & \multirow[t]{2}{*}{ Variable } & \multicolumn{4}{|c|}{ Time of measurement } \\
\hline & & to & t1 & t2 & t3 \\
\hline \multicolumn{6}{|c|}{ Assessment adolescents } \\
\hline YSR 11-18R & Mental health & $x$ & $x$ & $x$ & $x$ \\
\hline WHO-5 & Mental wellbeing & $x$ & $x$ & $x$ & $x$ \\
\hline KIDDI-SADS-PL & Mental health (interview) & $x$ & & & \\
\hline SES & Self-efficacy & $x$ & $x$ & $x$ & $x$ \\
\hline Brief-COPE & Coping strategies & $x$ & $x$ & $x$ & $x$ \\
\hline SD & Sociodemographic characteristics & $x$ & & & \\
\hline WAI-I & Working alliance & $x$ & $x$ & $x$ & $x$ \\
\hline OSSQ & Social support & $x$ & $x$ & $x$ & $x$ \\
\hline$E Q-5 D-Y$ & Health related life quality & $x$ & & & $x$ \\
\hline CAMSHRI-DE & Utilization of the health care system & $x$ & & & $x$ \\
\hline QO & Qualitative outcomes & & & & $x$ \\
\hline NEQ & Negative effects & & $x$ & $x$ & $x$ \\
\hline \multicolumn{6}{|c|}{ Assessment parent(s) and/or legal guardians } \\
\hline CBCL 6-18R & Proxy mental health of the adolescent & $x$ & & $x$ & $x$ \\
\hline M.I.N.I. & Mental health (interview) & $x$ & & & \\
\hline OSSQ & Proxy social support of the adolescent & $x$ & & $x$ & $x$ \\
\hline SD & Sociodemographic characteristics & $x$ & & & \\
\hline
\end{tabular}

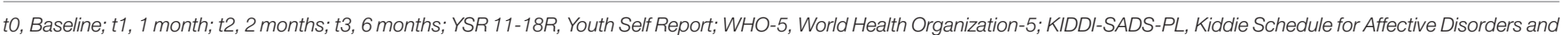

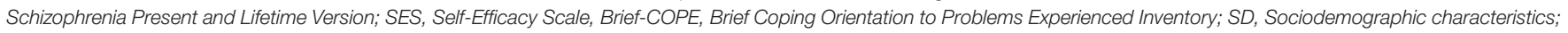

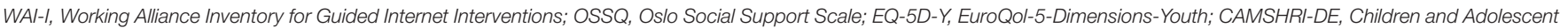

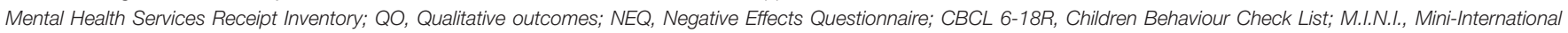
Neuropsychiatric Interview.

63 marks the cut-off to a clinically relevant score. Internal consistency of the full scale measured by Cronbach's $\alpha$ is $\geq 0.93$ (32).

\section{Secondary Outcomes Participants}

Mental health of the participant as measured by the Youth Self Report score (YSR 11-18R; 32). The 112 items can be divided into eight problem subscales (e.g., social problems or aggressive behaviors) and 2 second-level scales (internalizing and externalizing problems). All ten subscale scores will be calculated separately. For the eight problem subscales a tvalue above 70 marks the cut-off to a clinically relevant score, while for the internalizing and externalizing problems scales a $t$ value above 63 is considered clinically relevant. Internal consistency ranges for the subscales from $\alpha=0.59-0.84$ in a community sample and from $\alpha=0.62-0.90$ in a clinical sample (32).

Mental wellbeing of the participants will be measured with the World Health Organization-5 [WHO-5; (35)]. Internal consistency of this very economic scale with a processing time of under $1 \mathrm{~min}$ on average, assessed by Cronbach's $\alpha$ is $0.92(36)$.

Mental health of the participant as assessed by the German version (37) of the semi-structured clinical interview Kiddie Schedule for Affective Disorders and Schizophrenia Present and Lifetime Version [Kiddie-SADS-PL; (38)] designed to be used by trained interviewers to assess mental disorders according to DSM-IV and ICD-10 diagnostic criteria. For the interview fair to excellent interrater reliabilities for the different scales and for different languages have been reported $(39,40)$.

Self-efficacy of the participant as measured by the Self-Efficacy Scale [SES; (41)]. This 10 items scale has a good internal consistency, Cronbach's $\alpha$ between 0.8 and 0.9 (41).

Coping strategies of the participant as measured by the Brief Coping Orientation to Problems Experienced Inventory (42). The scale consists of 28 items which form 14 subscales, Cronbach's $\alpha$ for all scales range from $0.50-0.90$ (42).

\section{Secondary Outcomes Parent(s) and/or Legal Guardian(s)}

Proxy mental health of the participant at post-inclusion (t3) as measured by the Children Behaviour Check List (CBCL 6-18R; 32). This tool consists of 112 items, the items of the second part can be divided into eight subscales. All eight subscale scores separately and the full scale score will be calculated. For the eight subscales a t-value above 70 and for the full scale above 63 marks the cut-off to a clinically relevant score. Internal consistency of the full scale measured by Cronbach's $\alpha$ is $\geq 0.93$, while it ranges for the subscales from $\alpha=0.59-0.84$ in a community sample and from $\alpha=0.62-0.90$ in a clinical sample (32).

\section{Covariates}

The following variables will be included and assessed for their moderating or mediating role in the evaluation of the iCHIMPS intervention. 


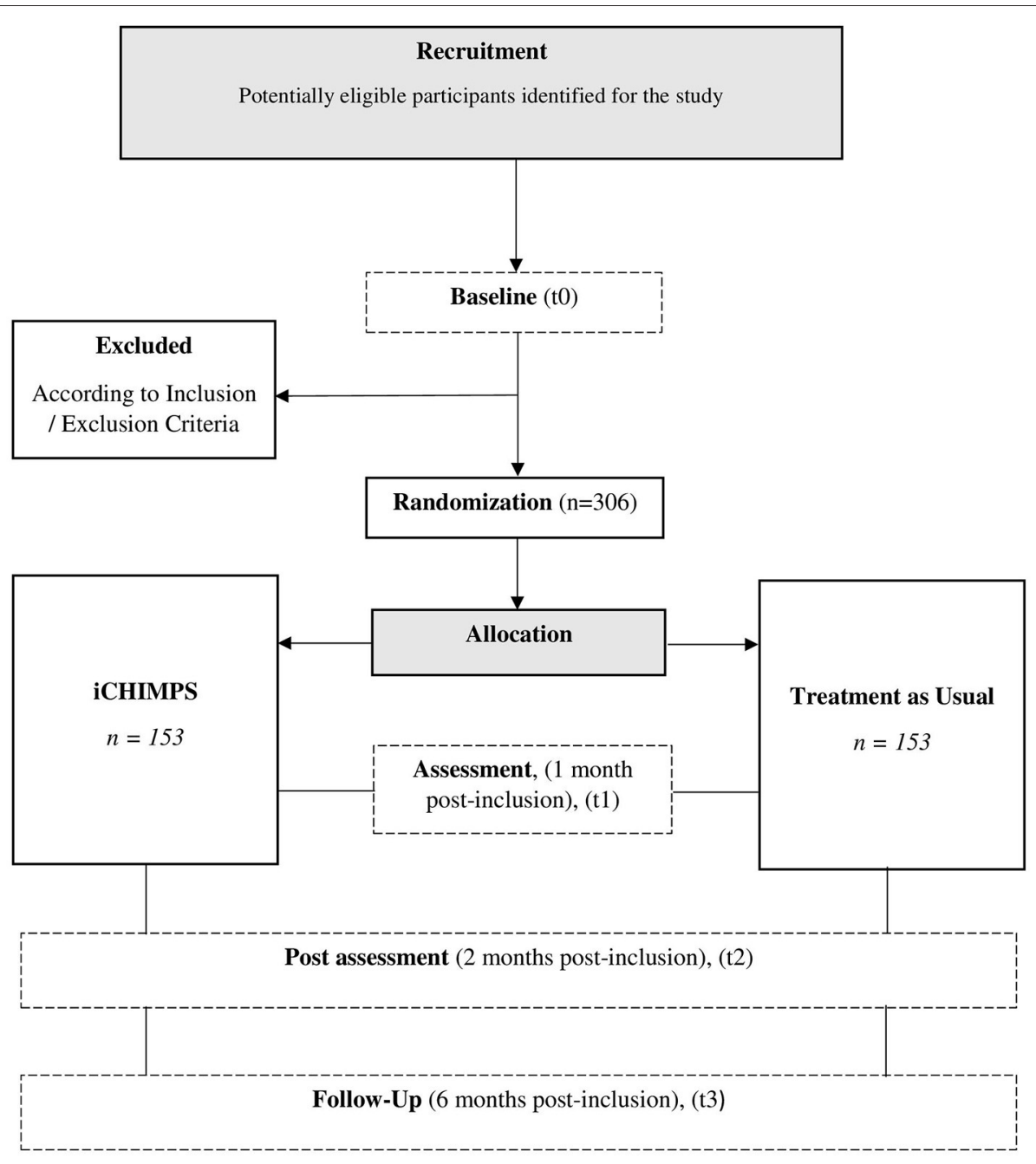

FIGURE 1 | Flow chart.

\section{Covariates Participants}

Participant characteristics as measured at baseline by selfreport items include age, gender, ethnicity, family composition, previous experiences with mental healthcare providers as well as educational and vocational background.

Working alliance between the participant and the E-Coach will be measured by the Working Alliance Inventory for Guided Internet Interventions [WAI-I, (43)]. This questionnaire is based on the Working Alliance Inventory (44) and was adapted for guided online interventions. The 12 items scale can be divided into 2 subscales and has a Cronbach's $\alpha$ of 0.93 for the whole scale, while the two subscales have $\alpha$ 's of 0.93 (task and goal agreement) and 0.89 (bond with therapist).

Social support of the participant as measured by the Oslo Social Support Scale 3 [OSSS-3; (45)]. The internal consistency for this short three item scale is 0.64 as measured by Cronbach's $\alpha(46)$.

\section{Covariates Parent(s) and/or Legal Guardian(s)}

Characteristics of the parent(s) or legal guardian(s) as measured by self-report items including age, gender, ethnicity, family composition, previous experiences with mental healthcare providers as well as educational and vocational background.

Mental health of the mentally ill parent(s) as assessed by the Mini-International Neuropsychiatric Interview [M.I.N.I.; (47)]. This tool was designed to be used by trained interviewers in a face to face setting to assess mental disorders according to DSM-IV and ICD-10 diagnostic criteria. Interrater reliabilities ranging from 0.43 to 0.82 for the different diagnoses have been reported for the interview (48).

Proxy social support of the participant as measured by the Oslo Social Support Scale 3 (OSSS-3; 45). The internal consistency of this short three item scale measured by Cronbach's $\alpha$ is 0.64 (46). 


\section{Qualitative Outcomes}

Open and closed questions concerning the adherence, acceptance, experiences, barriers, facilitators as well as potential drop-outs will be included into the online questionnaire.

\section{Economic Outcomes}

Health related life quality of the participant assessed by the self-report questionnaire EuroQol-5-Dimensions-Youth [EQ5D-Y; (49-52)]. The questionnaire consists of two parts. In the first part the participant defines his or her actual health state on the five dimensions mobility, self-care, usual activities, pain/discomfort and anxiety/depression. Each dimension is rated between 1 representing the best health state and 3 representing the worst health state. The overall health state is a 5-digit number representing the rating values for each health state. For the generation of QALYs, the overall health states will be valued using utility value sets generated by means of discrete choice experiments with representative samples from the general population $(53,54)$. In the absence of a national value set the visual analogue scale provided in the second part of the EQ-5D-Y can be used to value the overall health state.

Utilization of the health care system by the participant as measured by the self-report Children and Adolescent Mental Health Services Receipt Inventory (55, 56). This questionnaire assesses utilization of in- and outpatient health care as well as psychosocial care provided by the child welfare services and school based support provided by the educational system (56).

Health insurance claims data provided by one of the participating German health insurance companies, BARMER, DAK, KKH, TK, MKK, IKK Classic, AOK Baden-Württemberg or AOK Hessen, include information about the utilization of the health care system by members of the participating family who are insured by one of the aforementioned health insurance companies. The claims data consists of all treatment services provided by inpatient and outpatient care, medication, remedies, rehabilitation, and sick leave. All available information can be merged via an pseudonymized identification number for each patient. For every treatment service, all costs from health insurance perspective are documented for the period from January $1^{\text {st }} 2018$ to June $30^{\text {th }} 2022$.

\section{Negative Effects and Adverse Events}

(Serious) adverse events ((S)AEs) will be monitored and assessed in different ways through online questionnaires and structured interviews, adapted from the recommendations of the National Institute for Health Research (57) as well as from Horigian and colleagues (58).

(S)AEs can be reported to the E-Coaches during the intervention or to the study team via email or during the structured clinical interviews.

The deterioration rate will be one measure of possible negative effects of the iCHIMPS intervention. Deterioration is defined as worsening in the assessed mental health of the participant using the Reliable Change Index [RCI; $(59,60)]$.
Additionally, negative effects of psychological treatments experienced by the participant will be measured during the online assessments by the Negative Effects Questionnaire [NEQ; (61)]. The scale consists of 20 items and is divided into five subscales, namely symptoms, quality of treatment, dependency on the treatment, stigma, and hopelessness. The subscales are ranging in their internal consistency from Cronbach's $\alpha$ 0.72-0.93, while the whole scale shows a Cronbach's $\alpha$ of $0.94(61,62)$.

During the online self-report questionnaires (YSR; items 18 and 93) and during the structured clinical interviews (KiddieSADS-PL; depression chapter section 4 and 5) suicidal and selfinjurious tendencies and behaviors will be assessed.

\section{Completion Times}

The Kiddie-SADS-PL interview will take 45 to $75 \mathrm{~min}$ to administer and will be carried out at 0 with the participant and the parent(s) as well as at $\mathrm{t} 2$ and $\mathrm{t} 3$ with the participant.

The M.I.N.I. interview takes $\sim 15$ min to administer and will be carried out with the parents with a mental disorder at $t 0$.

Online self-report questionnaires for the participants will take $\sim 60$ min at $\mathrm{t} 0, \mathrm{t} 2$ and $\mathrm{t} 3$ as well as $\sim 30$ min at $\mathrm{t} 1$.

Online self-report questionnaires for the participating parents will take $\sim 20 \mathrm{~min}$ at $\mathrm{t} 0, \mathrm{t} 1, \mathrm{t} 2$ and $\mathrm{t} 3$.

\section{Reimbursement}

Only the participating adolescents will be compensated for their participation after the successful completion of the online assessment at $\mathrm{t} 2$ and $\mathrm{t} 3$ with each $20 €$. Reimbursement will be transferred to a bank account of their choice.

\section{Data Management, Quality Assurance, and Safety Measures}

All interviews will be conducted in person or via phone at the recruiting clinics and at the University Medical Center Hamburg-Eppendorf. The collected pseudonymized datasheets will be stored at the clinics until the end of each quarter and then sent to the study team of Ulm University. At Ulm University, the data will be stored and later transferred to the study team of the University Medical Center Hamburg-Eppendorf to be entered into the data management system provided by the data management company CTC North.

Online assessments will be carried out via the survey platform Lime Survey. The platform is based on servers located at Ulm University. All collected data will be stored pseudonymized. Data from the online assessments will be transferred to CTC North at the end of each quarter.

The CTC North will receive a list of all participants and/or their parents who are insured at one of the participating health insurance companies. Each cooperating health insurance company will then send the health insurance claims data from each participating family member who is a member of their insurance to CTC North.

At CTC North the data from all different sites will be collected and combined. Afterwards the evaluating partners will receive pseudonymized data sets for their respective analyses.

CTC North is additionally responsible for the monitoring of the data management measures at each recruiting clinic. 


\section{Safety Procedure of (S)AEs}

The occurrence of (S)AEs within the diagnostic interviews during the recruitment phase will be handled by the medical health professionals at each clinic, who also decide whether a study participation based on the inclusion and exclusion criteria is possible. Similarly, the occurrence of (S)AEs during the diagnostic interviews at $\mathrm{t} 3$, conducted by the study team at the University Medical Center Hamburg-Eppendorf, will be handled by medical health professionals of the local study team.

The occurrence of (S)AEs during the online assessment as well as during the internet-based intervention follows a step wise protocol and clear documentation rules and is handled by the study personnel at Ulm University. In case of (S)AEs, the participant will automatically receive an email with information on help as well as emergency numbers. The study personnel will contact participants who gave critical answers directly. If the participants are not reachable within $48 \mathrm{~h}$ on weekday the responsible authorities will be informed.

\section{Statistical Analysis Clinical Analyses}

All statistical analyses will be conducted according to the intention-to-treat principle (ITT). The ITT population includes all study participants of all families that have been randomized. Type 1 error rate (two-sided hypothesis) will be set to $5 \%$. Depending on the level of measurement of each variable descriptive statistics will be reported either for the whole sample or separated by groups.

Due to the fact that family members are subject to common influences, cluster effects are to be expected. Inferential statistics will therefore be conducted with a mixed-models approach (family and participant as random effects). Recruitment site, group membership, time point, and baseline value of each outcome as well as interaction between group membership and time point, as appropriate, will be added as covariates to the model. Endpoints will be operationalized as change from baseline value. Missing values will be direct imputed within the mixed model approach to enable an ITT analysis.

The result of the primary analysis is the between group contrast of the YSR total problems score at $\mathrm{t} 3$ (6-months postinclusion) within the ITT population. Only this comparison will be considered as confirmatory. Additionally, the analysis will be repeated with the per-protocol (PP) population. Secondary endpoints will be evaluated on an exploratory basis with similar models.

\section{Economic Evaluation}

The cost-effectiveness analysis follows the net benefit method (63-65). To estimate the incremental cost-effectiveness relation (ICER), the incremental costs for additional healthy life years (QALY) through the internet-based intervention will be determined. Cost information is derived from the CAMSHRI$\mathrm{DE}$ assessment tool and additionally from the resource use documented by the claims data. The stochastic uncertainties of the ICER will be estimated by non-parametric bootstrapping $(64,65)$. Interpretation of the results will be done on the basis of the cost-effectiveness acceptance curve $(64,65)$. The claims data analysis will be performed in accordance to the Good Practice of Secondary Data Analysis (GPS) guidelines (66).

\section{Moderator and Mediator Analyses}

Additional moderator and mediator as well as subgroup analyses will be carried out on an exploratory basis with the above defined covariates.

\section{DISCUSSION}

The iCHIMPS IMI, embedded into the multicenter project CHIMPS-NET, offers the opportunity to evaluate the clinicalas well as cost-effectiveness of an innovative form of a guided internet-based self-help intervention for children of parents with a mental disorder. Due to the high digital affinity of the younger generation, this digital mental health promotion offer might resonate especially well with the target audience. Insights from this study might enable us to establish online approaches as a viable mean to support children of parents with a mental disorder, thereby providing much needed mental health promotion offers in a time- and location-independent fashion to this often overlooked population.

The possibility to indirectly compare the iCHIMPS IMI, which is partly based on the CHIMPS-program, to the CHIMPS face-toface interventions, offers the opportunity to explore the benefits as well as short comings of each individual form of intervention delivery. This could increase the knowledge about when and possibly for whom which mode of delivery can offer advantages over the other, thereby paving the way for a discussion about a more flexible and patient centered approach to prevention and treatment interventions for the children and the whole family.

\section{Potential Problems and Solutions}

Potential problems during the trial might arise from data management, for example failing blinding, problems at data assessment, or storage. To prevent this from happening CHIMPS-NET is supported and supervised by the data management company CTC North. At CTC North the data from different sources will be collected, combined, and then send as pseudonymized data sheets to the evaluating project partners.

To avoid group contamination by participating siblings from the same family CHIMPS-NET has chosen to use a cluster randomized approach.

\section{CONCLUSION}

The iCHIMPS cRCT will examine the clinical- as well as cost-effectiveness of the internet-based iCHIMPS mental health promotion intervention for children of parents with mental disorders. This provides the opportunity to gain insights into an innovative as well as time- and location-independent form of support for this often overlooked at-risk group. Additionally, the larger CHIMPS-NET project allows indirect comparisons between online and face-to-face interventions for a similar target group. 


\section{TRIAL STATUS}

Recruitment has started on May $1^{\text {st }} 2021$.

\section{DISSEMINATION}

Final trial results are planned to be presented on national and international conferences and will be published in peerreviewed journals. Third party access to the final dataset might be provided depending on the agreement of data security and data exchange regulations.

\section{ETHICAL STATEMENT}

The present trial was approved by the Ethics Committee of the Ulm University (189/20-FSt/bal.). Additionally, every recruiting adult mental health clinic has obtained an individual approval by their local Ethics Committee in order to be able to recruit participants for this trial. All participating parties, in the case of children below the age of 16, and all legal guardians even if they themselves do not participate in any kind in the study, will have to sign written informed consent prior to any involvement.

\section{REFERENCES}

1. Mattejat F, Lisofsky B, editors. Nicht von schlechten Eltern: Kinder psychisch Kranker. Köln: BALANCE Buch + Medien Verlag (2014). p. 249.

2. Rasic D, Hajek T, Alda M, Uher R. Risk of mental illness in offspring of parents with schizophrenia, bipolar disorder, and major depressive disorder: a meta-analysis of family high-risk studies. Schizophr Bull. (2014) 40:2838. doi: 10.1093/schbul/sbt114

3. Wiegand-Grefe S, Geers P, Plaß A, Petermann F, Riedesser P. Kinder psychisch kranker Eltern: Zusammenhänge zwischen subjektiver elterlicher Beeinträchtigung und psychischer Auffälligkeit der Kinder aus Elternsicht. Kindheit und Entwicklung. (2009) 18:111-21. doi: 10.1026/0942-5403.18.2.111

4. Ashman SB, Dawson G, Panagiotides H. Trajectories of maternal depression over 7 years: Relations with child psychophysiology and behavior and role of contextual risks. Dev Psychopathol. (2008) 20:55-77. doi: 10.1017/S0954579408000035

5. Wiegand-Grefe S, Petermann F. Kinder psychisch erkrankter Eltern. Kindheit und Entwicklung. (2016) 25:63-7. doi: 10.1026/0942-5403/a000190

6. Potter R, Mars B, Eyre O, Legge S, Ford T, Sellers R, et al. Missed opportunities: mental disorder in children of parents with depression. Br J Gen Pract. (2012) 62:e487. doi: 10.3399/bjgp12X652355

7. Wiegand-Grefe S, Mattejat F, Lenz A, editors. Kinder mit psychisch kranken Eltern: Klinik und Forschung mit 55 Tabellen. Göttingen, Oakville: Vandenhoeck \& Ruprecht (2011). p. 496.

8. Zhou X, Zhang Y, Furukawa TA, Cuijpers P, Pu J, Weisz JR, et al. Different types and acceptability of psychotherapies for acute anxiety disorders in children and adolescents: a network meta-analysis. JAMA Psychiatry. (2019) 76:41-50. doi: 10.1001/jamapsychiatry.2018.3070

9. Battagliese G, Caccetta M, Luppino OI, Baglioni C, Cardi V, Mancini F, et al. Cognitive-behavioral therapy for externalizing disorders: a meta-analysis of treatment effectiveness. Behav Res Ther. (2015) 75:6071. doi: 10.1016/j.brat.2015.10.008

10. Reynolds S, Wilson C, Austin J, Hooper L. Effects of psychotherapy for anxiety in children and adolescents: a meta-analytic review. Clin Psychol Rev. (2012) 32:251-62. doi: 10.1016/j.cpr.2012.01.005

11. Zhou X, Teng T, Zhang Y, Del Giovane C, Furukawa TA, Weisz JR, et al. Comparative efficacy and acceptability of antidepressants,

\section{AUTHOR CONTRIBUTIONS}

SW-G, HB, RK, and JZ obtained funding for this study. HB is principle investigator of iCHIMPS. RK and JZ designed the health-economic evaluation and $\mathrm{AD}$ the statistical analysis. $\mathrm{PD}$ drafted the manuscript. All authors contributed to the final manuscript draft and approved the submitted version.

\section{FUNDING}

iCHIMPS as part of the larger multicenter study CHIMPS-NET is funded by the Innovation Committee (Innovationsausschuss) of the Joint Federal Committee in Germany (Gemeinsamer Bundesausschuss, GBA no: 01NVF18003).

\section{ACKNOWLEDGMENTS}

The authors would like to thank all CHIMPS-NET project members, all participating clinics, all participating health insurance companies, CTC North, all study teams of the individual subprojects, the developers of the eSano platform as well as all staff members and research assistants at Ulm University involved in designing and implementing iCHIMPS.

psychotherapies, and their combination for acute treatment of children and adolescents with depressive disorder: a systematic review and network metaanalysis. Lancet Psychiatry. (2020) 7:581-601. doi: 10.1016/S2215-0366(20) 30137-1

12. Cuijpers P. Targets and outcomes of psychotherapies for mental disorders: an overview. World Psychiatry. (2019) 18:276-85. doi: 10.1002/wps.2 0661

13. Siegenthaler E, Munder T, Egger M. Effect of preventive interventions in mentally ill parents on the mental health of the offspring: systematic review and meta-analysis. J Am Acad Child Adolesc Psychiatry. (2012) 51:817.e8. doi: 10.1016/j.jaac.2011.10.018

14. Kazdin AE. Addressing the treatment gap: a key challenge for extending evidence-based psychosocial interventions. Behav Res Ther. (2017) 88:718. doi: 10.1016/j.brat.2016.06.004

15. Kohn R, Saxena S, Levav I, Saraceno B. The treatment gap in mental health care. Bull World Health Organ. (2004) 82:858-66.

16. Levav I, Jacobsson L, Tsiantis J, Kolaitis G, Ponizovsky A. Psychiatric services and training for children and adolescents in Europe: results of a country survey. Eur Child Adolesc Psychiatry. (2004) 13:395401. doi: 10.1007/s00787-004-0427-6

17. Ebert DD, van Daele T, Nordgreen T, Karekla M, Compare A, Zarbo C, et al. Internet- and Mobile-Based Psychological Interventions: applications, efficacy, and potential for improving mental health. Eur Psychol. (2018) 23:167-87. doi: 10.1027/1016-9040/a000318

18. Domhardt $\mathrm{M}$, Steubl $\mathrm{L}$, Baumeister $\mathrm{H}$. Internet- and mobilebased interventions for mental and somatic conditions in children and adolescents. $Z$ Kinder Jugendpsychiatr Psychother. (2018) 48:33-46. doi: 10.1024/1422-4917/a000625

19. Baumeister H, Kraft R, Baumel A, Pryss R, Messner EM. "Persuasive E-Health Design for Behavior Change,". In: Baumeister H, Montag C, editors. Digital Phenotyping and Mobile Sensing: New Developments in Psychoinformatics. Cham: Springer International Publishing (2019). p. 261-76.

20. Baumeister H, Reichler L, Munzinger M, Lin J. The impact of guidance on Internet-based mental health interventions - a systematic review. Internet Intervent. (2014) 1:205-15. doi: 10.1016/j.invent.2014.08.003

21. Domhardt M, Schröder A, Geirhos A, Steubl L, Baumeister H. Efficacy of digital health interventions in youth with chronic 
medical conditions: a meta-analysis. Internet Intervent. (2021) 24:100373. doi: 10.1016/j.invent.2021.100373

22. Ebert DD, Zarski AC, Christensen H, Stikkelbroek Y, Cuijpers P, Berking $\mathrm{M}$, et al. Internet and computer-based cognitive behavioral therapy for anxiety and depression in youth: a meta-analysis of randomized controlled outcome trials. PLoS ONE. (2015) 10:e0119895. doi: 10.1371/journal.pone.01 19895

23. Reupert A, Bartholomew C, Cuff R, Foster K, Matar J, Maybery DJ, et al. An online intervention to promote mental health and wellbeing for young adults whose parents have mental illness and/or substance use problems: theoretical basis and intervention description. Front Psychiatry. (2019) 10:59. doi: 10.3389/fpsyt.2019.00059

24. Reupert A, Maybery D, Bartholomew C, Cuff R, Foster K, Matar J, et al. The acceptability and effectiveness of an online intervention for youth with parents with a mental illness and/or substance use issue. J Adolesc Health. (2020) 66:551-8. doi: 10.1016/j.jadohealth.2019.11.309

25. Woolderink M, Bindels JA, Evers SM, Paulus AT, van Asselt AD, van Schayck OC. An online health prevention intervention for youth with addicted or mentally ill parents: experiences and perspectives of participants and providers from a randomized controlled trial. J Med Internet Res. (2015) 17:e274. doi: 10.2196/jmir.4817

26. Wiegand-Grefe S, Halverscheid S, Plass A. Kinder und ihre psychisch kranken Eltern: Familienorientierte Prävention - Der CHIMPs-Beratungsansatz; mit CD-ROM. Göttingen u. a.: Hogrefe Verl. f. Psychologie (2011). p. 162.

27. Campbell MK, Piaggio G, Elbourne DR, Altman DG. Consort 2010 statement: extension to cluster randomised trials. BMJ Br Med J. (2012) 345:e5661. doi: 10.1136/bmj.e5661

28. Montgomery P, Grant S, Mayo-Wilson E, Macdonald G, Michie S, Hopewell S, et al. Reporting randomised trials of social and psychological interventions: the CONSORT-SPI 2018 Extension. Trials. (2018) 19:407. doi: 10.1186/s13063-018-2733-1

29. Husereau D, Drummond M, Petrou S, Carswell C, Moher D, Greenberg D, et al. Consolidated Health Economic Evaluation Reporting Standards (CHEERS) - explanation and elaboration: a report of the ISPOR health economic evaluation publication guidelines good reporting practices task force. Value in Health. (2013) 16:231-50. doi: 10.1016/j.jval.201 3.02.002

30. Ramsey SD, Willke RJ, Glick H, Reed SD, Augustovski F, Jonsson B, et al. Cost-effectiveness analysis alongside clinical trials II-An ISPOR good research practices task force report. Value Health. (2015) 18:16172. doi: 10.1016/j.jval.2015.02.001

31. Chan A-W, Tetzlaff JM, Gøtzsche PC, Altman DG, Mann H, Berlin JA, et al. SPIRIT 2013 explanation and elaboration: guidance for protocols of clinical trials. BMJ $\mathrm{Br}$ Med J. (2013) 346:e7586. doi: 10.1136/b mj.e7586

32. Döpfner M, Plück J, Kinnen C, Für die Arbeitsgruppe Deutsche Child Behavior Checklist. Manual Deutsche Schulalter-Formen der Child Behavior Checklist von Thomas, M. Achenbach. Elternfragebogen über das Verhalten von Kindern Und Jugendlichen (CBCL/6-18R), Lehrerfragebogen über das Verhalten von Kindern Und Jugendlichen (TRF/6-18R), Fragebogen für Jugendliche (YSR/11-18R); Hogrefe, Göttingen, Germany (2014).

33. Lunkenheimer F, Domhardt M, Geirhos A, Kilian R, Mueller-Stierlin AS, Holl RW, et al. Effectiveness and cost-effectiveness of guided Internet- and mobilebased CBT for adolescents and young adults with chronic somatic conditions and comorbid depression and anxiety symptoms (youthCOACHCD): study protocol for a multicentre randomized controlled trial. Trials. (2020) 21:253. doi: 10.1186/s13063-019-4041-9

34. Kraft R, Idrees AR, Stenzel L, Nguyen T, Reichert M, Pryss R, et al. eSano - An eHealth platform for internet-and mobile-based interventions. In: 2021 43rd Annual International Conference of the IEEE Engineering in Medicine and Biology Society (EMBC). IEEE (2021), 1997-2002.

35. Staehr JK. The use of well-being measures in primary health care-the DepCare project. World Health Organization, Regional Office for Europe: Well-Being Measures in Primary Health Care-the DepCare Project. Geneva: World Health Organization (1998).

36. Topp CW, Østergaard SD, Søndergaard S, Bech P. The WHO-5 Well-Being Index: a systematic review of the literature. Psychother Psychosom. (2015) 84:167-76. doi: 10.1159/000376585
37. Deutsche K-SADS-Arbeitsgruppe ICD-10, DSM III-R, DSM-IV Fassung der Kiddi-SADS-PL (5. Auflage der deutschen Forschungsversion, erweitert um ICD-10-Diagnostik). Klinik fuer Psychiatrie und Psychotherapie des Kindesund Jugendalt- ers, Frankfurt. (2001).

38. Kaufman J, Birmaher B, Brent D, Rao U, Flynn C, Moreci P, et al. Schedule for affective disorders and schizophrenia for school-age children-present and lifetime version (K-SADS-PL): initial reliability and validity data. J Am Acad Child Adolesc Psychiatry. (1997) 36:980-8. doi: 10.1097/00004583-19970700 $0-00021$

39. Ulloa RE, Ortiz S, Higuera F, Nogales I, Fresán A, Apiquian R, et al. Interrater reliability of the Spanish version of Schedule for Affective Disorders and Schizophrenia for School-Age Children-Present and Lifetime version (KSADS-PL). Actas Esp Psiquiatr. (2006) 34:36-40.

40. Lauth B, Magnusson P, Ferrari P, Pétursson H. An Icelandic version of the Kiddie-SADS-PL: translation, cross-cultural adaptation and inter-rater reliability. Nord J Psychiatry. (2008) 62:379-85. doi: 10.1080/08039480801984214

41. Schwarzer R, Jerusalem M. Skalen zur Erfassung von Lehrer- und Schülermerkmalen. Dokumentation der psychometri-schen Verfahren im Rahmen der Wissenschaftlichen Begleitung des Modellversuchs Selbstwirksame Schulen. Berlin: Freie Universität Berlin (1999).

42. Carver CS. You want to measure coping but your protocol's too long: consider the brief COPE. Int J Behav Med. (1997) 4:92-100. doi: 10.1207/s15327558ijbm0401_6

43. Gómez Penedo JM, Berger T. grosse Holtforth M, Krieger T, Schröder J, Hohagen F, et al. The working alliance inventory for guided internet interventions (WAI-I). J Clin Psychol. (2020) 76:973-86. doi: 10.1002/jclp.22823

44. Munder T, Wilmers F, Leonhart R, Linster HW, Barth J. Working alliance inventory-short revised (WAI-SR): psychometric properties in outpatients and inpatients. Clin Psychol Psychother. (2010) 17:231-9. doi: 10.1002/cpp.658

45. Dalgard OS, Dowrick C, Lehtinen V, Vazquez-Barquero JL, Casey P, Wilkinson G, et al. Negative life events, social support and gender difference in depression. Soc Psychiatry Psychiatr Epidemiol. (2006) 41:44451. doi: 10.1007/s00127-006-0051-5

46. Kocalevent RD, Berg L, Beutel ME, Hinz A, Zenger M, Härter M, et al. Social support in the general population: standardization of the Oslo social support scale (OSSS-3). BMC Psychol. (2018) 6:31. doi: 10.1186/s40359-018-0249-9

47. Sheehan DV, Lecrubier Y, Sheehan KH, Amorim P, Janavs J, Weiller E, et al. The Mini-International Neuropsychiatric Interview (MINI): the development and validation of a structured diagnostic psychiatric interview for DSM-IV and ICD-10. J Clin Psychiatry. (1998) 59:22-33. doi: 10.1037/t18597-000

48. Lecrubier Y, Sheehan DV, Weiller E, Amorim P, Bonora I, Harnett Sheehan $\mathrm{K}$, et al. The Mini International Neuropsychiatric Interview (MINI). A short diagnostic structured interview: reliability and validity according to the CIDI. Eur Psychiatry. (1997) 12:224-31. doi: 10.1016/S0924-9338(97)83296-8

49. Kreimeier S, Åström M, Burström K, Egmar AC, Gusi N, Herdman $M$, et al. EQ-5D-Y-5L: developing a revised EQ-5D$\mathrm{Y}$ with increased response categories. Qual Life Res. (2019) 28:1951-61. doi: 10.1007/s11136-019-02115-X

50. Kreimeier S, Greiner W. EQ-5D-Y as a health-related quality of life instrument for children and adolescents: the instrument's characteristics, development, current use, and challenges of developing its value set. Value Health. (2019) 22:31-7. doi: 10.1016/j.jval.2018.11.001

51. Shah KK, Ramos-Goñi JM, Kreimeier S, Devlin NJ. An exploration of methods for obtaining $0=$ dead anchors for latent scale EQ-5D-Y values. Eur J Health Econ. (2020) 21:1091-103. doi: 10.1007/s10198-020-01205-9

52. Wille N, Badia X, Bonsel G, Burström K, Cavrini G, Devlin N, et al. Development of the EQ-5D-Y: a child-friendly version of the EQ-5D. Qual Life Res. (2010) 19:875-86. doi: 10.1007/s11136-010-9648-y

53. Ramos-Goñi JM, Oppe M, Stolk E, Shah K, Kreimeier S, Rivero-Arias O, et al. International Valuation Protocol for the EQ-5D-Y-3L. Pharmacoeconomics. (2020) 38:653-63. doi: 10.1007/s40273-020-00909-3

54. Kreimeier S, Oppe M, Ramos-Goñi JM, Cole A, Devlin N, Herdman M, et al. Valuation of EuroQol Five-Dimensional Questionnaire, Youth Version (EQ5D-Y) and EuroQol Five-Dimensional Questionnaire, Three-Level Version (EQ-5D-3L) Health States: the impact of wording and perspective. Value Health. (2018) 21:1291-8. doi: 10.1016/j.jval.2018.05.002 
55. Kilian R, Losert C, McDaid D, Park A, Knapp M, Beecham J, et al. The health economic evaluation of children and adolescent mental health services across the enlarged Europe. Ulm: Ulm University, Department of Psychiatry and Psychotherapy II (2009).

56. Waldmann T, Stiawa M, Dinc Ü, Saglam G, Busmann M, Daubmann A, et al. Costs of health and social services use in children of parents with mental illness. Child Adolesc Psychiatry Ment Health. (2021) 15:10. doi: 10.1186/s13034-021-00360-y

57. Duggan C, Parry G, McMurran M, Davidson K, Dennis J. The recording of adverse events from psychological treatments in clinical trials: evidence from a review of NIHR-funded trials. Trials. (2014) 15:335. doi: 10.1186/1745-6215-15-335

58. Horigian VE, Robbins MS, Dominguez R, Ucha J, Rosa CL. Principles for defining adverse events in behavioral intervention research: lessons from a family-focused adolescent drug abuse trial. Clin Trials. (2010) 7:5868. doi: 10.1177/1740774509356575

59. Jacobson NS, Follette WC, Revenstorf D. Psychotherapy outcome research: methods for reporting variability and evaluating clinical significance. Behav Ther. (1984) 15:336-52. doi: 10.1016/S0005-7894(84)80002-7

60. Evans C, Margison F, Barkham M. The contribution of reliable and clinically significant change methods to evidence-based mental health. Evid Based Ment Health. (1998) 1:70. doi: 10.1136/ebmh.1.3.70

61. Rozental A, Kottorp A, Boettcher J, Andersson G, Carlbring P. Negative effects of psychological treatments: an exploratory factor analysis of the negative effects questionnaire for monitoring and reporting adverse and unwanted events. PLoS ONE. (2016) 11:e0157503. doi: 10.1371/journal.pone.0157503

62. Rozental A, Kottorp A, Forsström D, Månsson K, Boettcher J, Andersson G, et al. The Negative Effects Questionnaire: psychometric properties of an instrument for assessing negative effects in psychological treatments. Behav Cogn Psychother. (2019) 47:559-72. doi: 10.1017/S1352465819000018

63. Glick HA, Doshi JA, Sonnad SS, Polsky D. Economic Evaluation in Clinical Trials. New York, NY: OUP Oxford (2014).

64. Salize HJ, Kilian R. Gesundheitsökonomie in der Psychiatrie: Konzepte, Methoden, Analysen. Stuttgart, Kohlhammer Verlag (2010).
65. Willan AR, Briggs AH. Statistical Analysis of Cost-Effectiveness Data. Chichester: John Wiley \& Sons (2006).

66. Swart E, Gothe H, Geyer S, Jaunzeme J, Maier B, Grobe TG, et al. Gute Praxis Sekundärdatenanalyse (GPS): Leitlinien und Empfehlungen. Das Gesundheitswesen. (2015) 77:120-6. doi: 10.1055/s-0034-1396815

Conflict of Interest: The study team of the Ulm University are authors of this manuscript and have developed the internet and mobile-based intervention iCHIMPS. Hence, the final statistical analysis will be conducted by independent evaluators from Ulm University and the Center for Health Economic Research in Hannover (CHERH). HB received consultancy fees, reimbursement of congress attendance and travel costs as well as payments for lectures from Psychotherapy and Psychiatry Associations as well as Psychotherapy Training Institutes in the context of E-Mental-Health topics. He has been the beneficiary of study support (third-party funding) from several public funding organizations.

The remaining authors declare that the research was conducted in the absence of any commercial or financial relationships that could be construed as a potential conflict of interest.

Publisher's Note: All claims expressed in this article are solely those of the authors and do not necessarily represent those of their affiliated organizations, or those of the publisher, the editors and the reviewers. Any product that may be evaluated in this article, or claim that may be made by its manufacturer, is not guaranteed or endorsed by the publisher.

Copyright (c) 2022 Dülsen, Barck, Daubmann, Höller, Zeidler, Kilian, WiegandGrefe and Baumeister. This is an open-access article distributed under the terms of the Creative Commons Attribution License (CC BY). The use, distribution or reproduction in other forums is permitted, provided the original author(s) and the copyright owner(s) are credited and that the original publication in this journal is cited, in accordance with accepted academic practice. No use, distribution or reproduction is permitted which does not comply with these terms. 